\title{
How to establish an academic SARS-CoV-2 testing laboratory
}

To the Editor - To meet the demands for SARS-CoV-2 testing capacity in the United Kingdom, a network of laboratories was set up from March 2020 onwards. We document our experience and learnings from implementing an automated, medium-throughput testing facility for SARS-CoV-2 in the University of Birmingham Turnkey project.

The incursion of SARS-CoV-2 into the United Kingdom in February and March 2020 (ref. ${ }^{1}$ ) led to an enormous surge in COVID-19 cases, with ensuing hospitalisations and deaths from April through to the end of July. On 11 March 2020, the Health Secretary announced plans for the scaling of COVID-19 testing, and on 27 March 2020 it was announced at the daily national coronavirus briefing ${ }^{2}$ that a new alliance of private sector and academic collaborative laboratories were being created to generate the extra testing capacity required. This radical plan moved SARS-CoV-2 diagnostics out of the national clinical laboratory and Public Health England infrastructure, and into a network of massive-throughput laboratories dedicated to COVID-19 testing, named 'lighthouse laboratories'. These facilities were developed to provide the processing capacity of up to 100,000 tests in 24 hours by the end of April, with all tests belonging to the so-called 'Pillar 2' programme of testing individuals with symptomatic COVID-19 in the community ${ }^{3}$.

As testing ramped up and we moved into summer, the R number dropped below 1 . Then, in September 2020, a resurgence in COVID-19 cases in the United Kingdom occurred in parallel with the onset of cooler weather and seasonal respiratory infections such as rhinovirus ${ }^{4}$. Without testing, it is not possible to differentiate between infection with COVID-19 and seasonal respiratory infections because early symptoms of both are overlapping. Owing to this there was a surge in demand for SARS-CoV-2 testing which overwhelmed the national Pillar 2 testing infrastructure.

To further extend testing capacity, government officials from the Department of Health and Social Care (DHSC) have identified national expertise in Higher Education establishments and private laboratories. In response to a request to set up a testing laboratory, we established the Birmingham Turnkey facility, a medium-throughput COVID-19 testing facility at the University of Birmingham that operates within the United Kingdom's national Pillar 2 testing infrastructure and is capable of processing 3,800 tests in 12 hours. Establishing our laboratory took three months from those initial conversations.

Our laboratory differs from other academic testing facilities that have been set up around the world ${ }^{5,6}$; it has been established in collaboration with the DHSC to ensure that the results feed directly into the national Pillar 2 infrastructure, rather than being a 'stand-alone service'.

Here, we describe the modular design of our test laboratory, including methods we used to enable automation of the testing process, and how we handle data flow and the quality and reporting of results. We provide our entire audit documentation (https://doi.org/10.6084/ m9.figshare.13096238.v2; ref. ${ }^{7}$ ), including laboratory protocols, and hope that our experiences and the materials we provide in Figshare help others to set up rapid-response diagnostic centres in academic laboratories around the world.

\section{Modular and transferable laboratory set-up}

The Birmingham Turnkey facility divides the end-to-end diagnostic process into discrete modules, and was based on the design implemented in the Milton Keynes lighthouse laboratory hosted by the UK Biosample Centre (A.M. was the infectious disease lead in the implementation of this laboratory). The laboratory's modular design can be scaled up or down as needed by increasing or decreasing the number of modules. Each module is in a separate area, thereby reducing the risk of reagent contamination throughout the process (Fig. 1).

Samples are received in module 1 as swabs in a screw-capped viral transport medium tube which is sealed in a MedDX FS95 safety bag. The tube and bag are already barcoded using labels assigned at the testing centre or in the home test kit. The bags are collated in sets of 94 in a plastic tray and placed in an oven (Memmert UN110) set to a temperature of $56^{\circ} \mathrm{C}$. Samples are left for 90 minutes to allow the viral transport medium (VTM) to reach the validated range of $56^{\circ} \mathrm{C}$ for 30 minutes, which is shown to result in a 5 - $\log$ reduction in cultivable virus particles $^{8}$. This heat inactivation step is quality controlled using a thermometer in a sample tube inside the oven to ensure the correct temperature is reached. The samples are now considered sufficiently low of infectious hazard to perform diagnostic testing within containment level 2 (ref. ${ }^{9}$ ) and are transported to module 2 , which is a processing laboratory in a separate room where samples are unbagged and placed on racks compatible with the automated liquid-handling equipment of choice (in our case, the Tecan Freedom Evo150). Any samples that have leaked are transported to a class II microbiological safety cabinet for safe manual processing.

Module 3 is an automated liquid-handling platform (we use a Tecan Freedom Ev0150; see Fig. 1) which can process 282 samples at a time. Sample tubes are manually uncapped inside the robot work station and the automated process is initiated, the first part of which is the scanning of the sample tube's barcode into our laboratory information management system (LIMS) using a rapid inbuilt scanner on the Tecan platform (we use a Thermofisher LIMS system easily compatible with our automated liquid handlers and quantitative PCR (qPCR) platforms). Automated dispensing of the VTM from the sample tube into a 96-well plate enables RNA extraction. We use MagMax RNA extraction reagents from ThermoFisher, so our VTM sample is pipetted into a 96-well plate containing MagMax viral binding buffer, which also acts as a lysis solution and renders the plate safe to use at containment level 2. The MagMax magnetic bead solution can also be added by the automated liquid handler, but we prefer manual addition using a multichannel pipette as this frees up the liquid-handling platform to process more samples in a defined time period. This means that an additional 4-5 plates can be processed in a 12-hour shift. To each well (except the negative control), we also pipette an internal MS2 phage control that comes with our PCR assay of choice, which acts as an extraction and amplification control for each sample tested. The time for a 96-well plate to be processed in module 
3 is 20 minutes. For RNA extraction in module 4 we use the MagMax nucleic acid extraction system; after all samples and beads have been added to the plate, we use the Kingfisher automated nucleic acid extraction platform with the associated MagMax reagents and plates. Our system operates two Kingfisher units. Once RNA is eluted into the 96-well elution buffer plate, the plate is sealed with a foil and taken to module 5. The time for a 96-well plate to be processed in module 4 is 25 minutes.

Another automated liquid-handling platform (the Tecan Freedom EVO 75) is used in module 5 to set up the 96 -well qPCR plate. We have chosen to use the Thermo TaqPath COVID-19 multiplex assay, which amplifies the open reading frame (orf) $1 a / b$ junction and the $\operatorname{orf} N$ and $\operatorname{orf} S$ genes of the SARS-CoV-2 genome, and also includes an internal MS2 extraction and amplification phage control for each well. The master mix, primer mix and water are mixed manually in a 15-ml falcon tube and then dispensed into a pipetting trough. The liquid handler is initiated and the PCR mix is pipetted into each well of a 96-well optical PCR plate. The RNA is then pipetted into the PCR plate (kept on a cooling unit in the robot) and an optical film seal applied, before being taken to module 6 . The time for a 96 -well plate to be processed in module 5 is 10 minutes.

Module 6 is comprised of a separate room housing six qPCR platforms. In our case, the PCR machine must be compatible with the TaqPath assay and we therefore use the QuantStudio 5 platform. The assay-specific qPCR run takes around 60 minutes. Following this, the run data are analysed using proprietary ThermoFisher COVID analysis software that allows for the automation of results for each well according to performance of the internal MS2 phage control run dynamics.

\section{Staffing, capacity, costs and turnaround times}

Our six modules enable $40 \times 96$-well plates to be processed in a 12 -hour day, staffed by 12 people. This equates to around 3,800 samples per 12-hour shift. In lighthouse laboratories like the one in Milton Keynes, there are 5 modular systems which are combined to analyse 200 plates per 12-hour shift and operate 24 hours per day, 7 days per week, with 60 staff per shift. Most staff work in the unbagging and heat inactivation modules, which are the most labour-intensive and time-limiting stage of any diagnostic process involving a high-containment-level pathogen.

In the Turnkey facility we have eight staff per shift working in modules 1 and
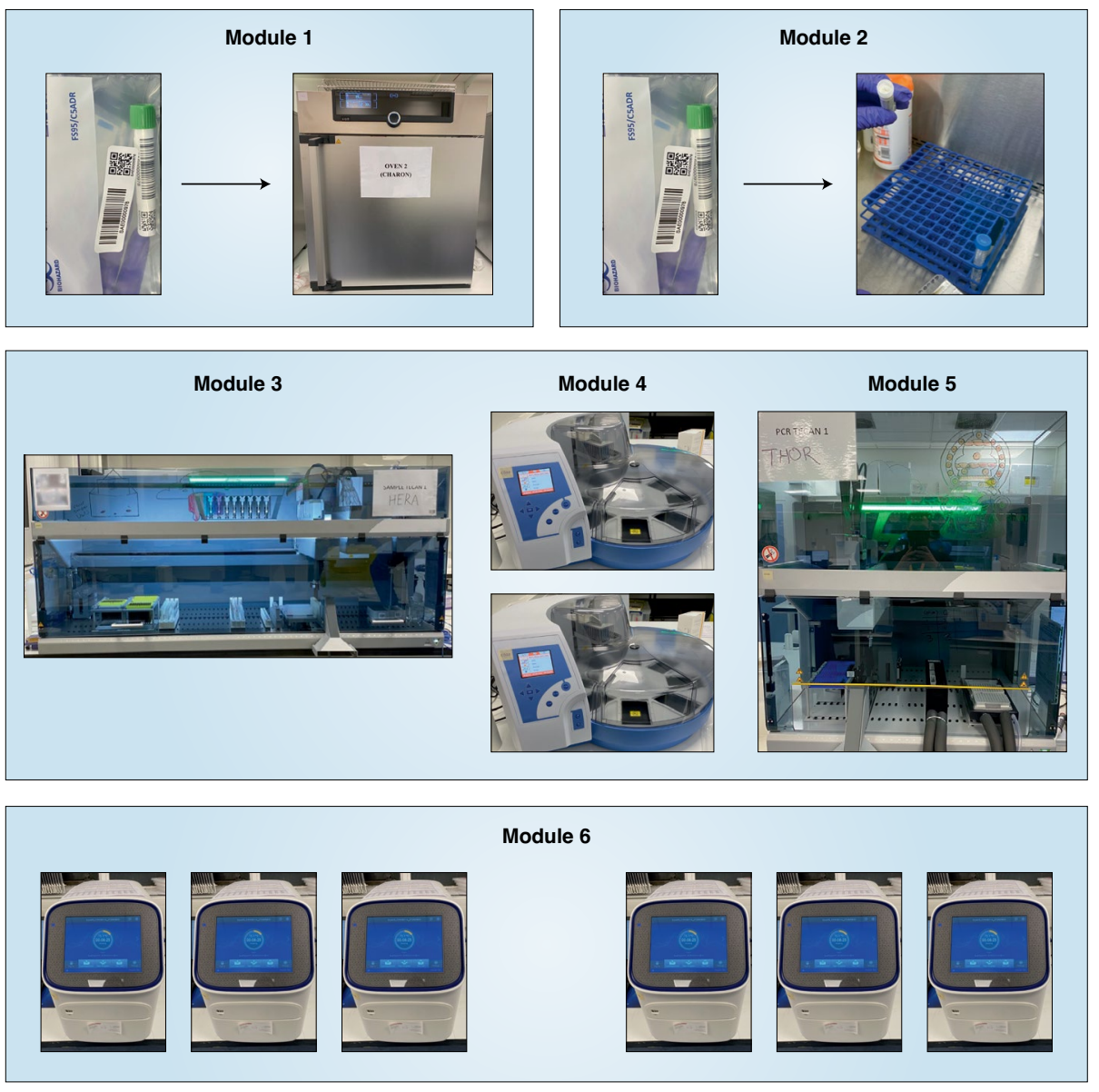

Fig. 1 | A Turnkey lab modular set up. Module 1 is housed in a dedicated room for heat inactivation of samples. Module 2 is also in a dedicated room where inactivated samples are unbagged and racked for automated processing. Modules 3, 4 and 5 are housed together in a laboratory. The initial liquid-handling platform in module 3 aliquots samples into a 96-well plate, which is then used to perform automated magnetic-bead based RNA extraction in module 4. A subsequent liquid-handling platform in module 5 prepares a 96-well PCR plate which is analysed on a suitable QPCR machine in module 6 within a stand-alone room. This complete module is sufficient to process around 3,800 swabs per 12-hour shift. Photographs courtesy of Alan McNally.

2, two staff members for modules 3 and 4 , and two staff members for modules 5 and 6 . We use 2 complete shift teams who work 12-hour days on a rolling schedule of 4 days on and 4 days off. From heat inactivation to the end of $\mathrm{qPCR}$, the turnaround time for a 96-well plate is approximately $3-3.5$ hours. The cost per test for reagents in our workflow equates to $£ 8.41$ per test, calculated on buying reagents to perform 3,800 tests per day, every day, for 6 months. Based on that same proviso, factoring in the shift costs of 8 staff members on a band-500 salary and 4 staff members on a level-7 salary, and the full economic costing of senior managerial staff, plus offsetting the costs of all equipment and IT systems across the 6 months, the complete cost per test equates to approximately $£ 18$ per test.

\section{Pathway to success at Turnkey}

Establishing a testing laboratory for a notifiable infectious disease using the accredited framework of the NHS and Public Health England is not straightforward. The Birmingham Turnkey laboratory was only possible due to the existing expertise of the University's Clinical Immunology Service, which has delivered ISO15189-accredited diagnostics on behalf of the NHS for many years. Using this existing framework has allowed us to meet the exacting standards required for a Pillar 2 testing laboratory. Before testing can begin, a full national testing laboratory audit report ( ref. $^{7}$ ) needs to be generated for the laboratory working towards UKAS accreditation. This ensures that each laboratory operates to the same standards as Public Health England's diagnostic laboratories, with validated 
standard operating procedures for each process in the diagnostic chain, full training records, emergency contingencies, quality management and clear governance from appropriately qualified personnel.

Key to the success of the Turnkey laboratory is a close working relationship with our local NHS trust. We believe independent clinical oversight of our testing is crucial to maintain trust in Pillar 2 COVID-19 testing and to provide assurance that the testing we do is to the highest possible standard. We work with our partners in University Hospital Birmingham NHS Foundation Trust, and we have a seconded clinical virologist and clinical virology scientist from the same trust to work with us for $\sim 3$ days per week. This provides us with clinical virology expertise and oversight of the quality of data that we generate. To facilitate data flow in the laboratory we purchased a dedicated LIMS system for the facility, which tracks samples through the end-to-end workflow and generates a .CSV results file containing the sample's barcode, Ct values for each of the three target genes in the PCR assay, and a result of 'positive', 'negative' or

'void'. This results file is double-checked by our clinical colleagues, or by a member of the laboratory team they have personally trained and signed off as competent, and is then uploaded to the National Pathology Exchange system (which is used for clinical result management in the NHS) every hour through our LIMS system.

Validation of our processes includes testing the performance characteristics of each piece of equipment and the precision and accuracy of the whole end-to end process during set up and validation, and then in ongoing monthly NEQAS validation tests. In addition, we established the detection limits, linearity, intra- and inter-assay variations, stability of samples following heat extraction, performance of the LIMS and interpretive software, and excluded carry-over and plate effects. This was done by using the Qnostics SARS-CoV-2 analytic panel ${ }^{10}$ and un-extracted and RNA-extracted clinical samples from another laboratory that had been previously approved through the national testing laboratory audit report. For ongoing internal quality control material, we use the Qnostics SARS-CoV-2 positive control (https://www.randox.com/sars-cov2-molecular-control/) in the 96th well of every plate, which should have a Ct value of approximately 25 . This acts as a control for both our RNA extraction and PCR amplification. The distribution of $\mathrm{Ct}$ values is established over 10 complete runs of the assay and is plotted on Levey-Jennings charts, and the primary samples are reanalysed if the positive control is 2 standard deviations outside of the expected $\mathrm{Ct}$ value. In addition, a positive pooled sample has been established to monitor the Qnostic positive control.

Turnkey means 'the provision of a complete product or service that is ready for immediate use'. We benefited from the experiences of A.M., who helped set up the lighthouse laboratory in Milton Keynes, and M.M., O.M., T.W., J.M. and B.P., who all worked in a lighthouse laboratory, with regards to the methods, equipment and processes described. However, the Birmingham Turnkey was not a 'drag and drop' laboratory, and we anticipate the same will apply to other sentinel laboratories. Setting up the facility within the three-month time frame agreed with the DHSC required enormous help from colleagues across the university. A dedicated laboratory space suitable for sample delivery and processing logistics had to be recommissioned in around six weeks. Colleagues from finance played a key role by costing the project and setting up rolling purchase orders with key suppliers. Colleagues from legal services created a contract with the DHSC for us to provide Pillar 2 testing at agreed costs in a very short time scale, and human-resources colleagues worked tirelessly to recruit staff on short-term contracts for the facility in very little time.

The key requirement to establishing the facility was to work hand-in-hand with local clinical diagnostic scientists. We advise that such partnerships are crucial if academic researchers are to set up the efficient and effective diagnostic laboratories that are now needed to cope with the anticipated increases in demand for COVID-19 tests in the winter of 2020 and beyond.
Alex Richter', Tim Plant', Michael Kidd², Andrew Bosworth ${ }^{3}$, Megan Mayhew', Oliver Megram?, Fiona Ashworth', Liam Crawford (D)', Thomas White', Emma Moles-Garcia', Jeremy Mirza', Benita Percival ${ }^{1}$ and Alan McNally (D) 4 四 ${ }^{1}$ Clinical Immunology Service, Institute of Immunology and Immunotherapy, College of Medical and Dental Science, University of Birmingham, Birmingham, UK. ${ }^{2}$ Public Health England, Birmingham, UK. ${ }^{3}$ University Hospital Birmingham NHS Foundation Trust, Birmingham, UK. ${ }^{4}$ Institute of Microbiology and Infection, College of Medical and Dental Science, University of Birmingham, Birmingham, UK.

$\bigotimes_{\text {e-mail:a.monally.1@bham.ac.uk }}$

Published online: 2 November 2020 https://doi.org/10.1038/s41564-020-00818-3

References

1. Pybus, O. et al. Preliminary analysis of SARS-CoV-2 importation \& establishment of UK transmission lineages. Virological. org https://virological.org/t/preliminary-analysis-of-sars-cov2-importation-establishment-of-uk-transmission-lineages/507 (2020).

2. Gove, M. Chancellor of the Duchy of Lancaster statement on coronavirus (COVID-19): 27 March 2020. GOV.UK https://www.gov.uk/government/speeches/ chancellor-of-the-duchy-of-lancaster-statement-oncoronavirus-covid-19-27-march-2020 (2020).

3. Coronavirus (COVID-19): Scaling Up Our Testing Programmes (Department of Health and Social Care, 2020).

4. PHE National Influenza Report: Summary of UK Surveillance of Influenza and Other Seasonal Respiratory Illnesses, 17 September 2020 - Week 38 Report (Public Health England, 2020).

5. Amen, A. M. et al. Nat. Biotechnol. 38, 791-797 (2020).

6. Berger Gillam, T. et al. Preprint at medXriv https://doi. org/10.1101/2020.09.22.20199455 (2020).

7. McNally, A. Birmingham Turnkey national audit for publication. Figshare https://doi.org/10.6084/ m9.figshare.13096238.v2 (2020).

8. SARS-CoV-2 Inactivation Testing: Interim Report (Public Health England, 2020); https://assets.publishing.service.gov. uk/government/uploads/system/uploads/attachment_data/ file/905783/HCM-CoV2-009-v3_Heat_Treatment_TCF-1.pdf

9. Position statement on inactivation of SARS-CoV-2: Implications for laboratory testing. Public Health England https://www.gov.uk/government/publications/ position-statement-regarding-covid-19-tests-evaluated-by-phe/ position-statement-on-inactivation-of-sars-cov2-implications-for-laboratory-testing (2020). 10. SARS-CoV-2 Analytical Q Panel 01. Qnostics https://www.qnostics.com/wp-content/uploads/2020/05/ SCV2AQP01-A-RBPL3155-Rev01.pdf (2020).

\section{Author contributions}

A.M. wrote the manuscript. All authors planned, designed and implemented the Turnkey laboratory. All authors contributed to the revisions and final draft of the manuscript.

\section{Competing interests}

The authors declare no competing interests. 